\title{
1 Gut Microbiome Biomarkers in Adolescent Obesity: a Regional
}

\section{Study}

3 Xuefeng $\mathrm{Gao}^{1,2+}$, Binbin $\mathrm{Wu}^{3,4+}$, Yonglong $\mathrm{Pan}^{3}$, Shaoming Zhou ${ }^{5}$, Ming Zhang ${ }^{6}$,

4 Yunpeng $\mathrm{Cai}^{7^{*}}$, Yan Liang $^{8^{*}}$

$6{ }^{1}$ Department of gastroenterology and hepatology, Shenzhen University General Hospital, Shenzhen, China

$7 \quad 2$ Shenzhen University Clinical Medical Academy, Shenzhen, China

$8{ }^{3}$ Institute of Biomedicine and Biotechnology, Shenzhen Institutes of Advanced Technology, Chinese Academy of

9 Sciences, Shenzhen, China

104 University of Chinese Academy of Sciences, Beijing, China

115 Department of Gastroenterology, Shenzhen Children's Hospital, Shenzhen, China

$12{ }^{6}$ Department of Nutrition, Peking University Shenzhen Hospital, Shenzhen, China

$13{ }^{7}$ Research Center for Biomedical Information Technology, Shenzhen Institutes of Advanced Technologies, Chinese

14 Academy of Sciences, Shenzhen, China

$15{ }^{8}$ School of Resources and Environment, University of Electronic Science and Technology of China, Chengdu, China

16 NO. OF WORDS: 4,649

$17{ }^{+}$Equal contribution

18 * Correspondence:

19 Yunpeng Cai, E-mail: yp.cai@siat.ac.cn

20 Yan Liang, E-mail: yliang@uestc.edu.cn 


\section{ABSTRACT}

22 Purpose: This study aimed to characterize the gut microbiota in obese Shenzhen

23 adolescents, and evaluate the influence of gender on BMI-related differences in the gut

24 microbiome.

25 Methods: Physical examinations, blood pressure measurement, serological assay, and body composition evaluation were conducted on two-hundred and five adolescents from Shenzhen. Fecal microbiome composition was profiled via 16S rRNA gene sequencing. A Random Forest (RF) classifier model was built to distinguish the BMI categories based on the gut bacterial composition.

Results: Fifty-six taxa consisting mainly of Firmicutes were identified that having

31 significant associations with BMI; two OTUs belonging to Ruminococcaceae and one belonging to Lachnospiraceae had relatively strong positive correlations with body fate rate, waistline, and most of serum biochemical parameters. Based on the $56 \mathrm{BMI}-$ for predicting the obese phenotype. Gender-specific differences in the gut microbiome composition was obtained, and a lower relative abundance of Odoribacter was particularly found in obese boys. Functional analysis revealed a deficiency in bacterial gene contents related to PPAR signaling pathway in obese subjects for both genders; significantly lower levels of adipocytokine signaling pathway and ethylbenzene degradation were particularly detected in obese girls.

41 Conclusions: This study revealed unique features of gut microbiome in terms of microbial composition and metabolic functions in obese Shenzhen adolescents. The 
43 effect of geographical location, age and gender on the gut microbiome should be

44 carefully considered in case-control studies.

45 Key Words: adolescent obesity; feces; gut microbiota; 16S rRNA; signaling pathway 46 


\section{INTRODUCTION}

Obesity during childhood and adolescence is associated with cardiovascular disease and metabolic syndrome later in life, and has become a significant public health concern worldwide (Collaboration NCDRF. 2017). The human gut microbiome is being increasingly recognized as an important factor in the development of obesity (Ley et al. 2006; Shen et al. 2013), and it is highly involved in the host calorie harvest and energy homeostasis (Rios-Covian et al. 2016). Alterations in the gut microbiota caused by such as antibiotics exposure, is linked to a variety of metabolic diseases including obesity, type 1 and type 2 diabetes (Cox and Blaser. 2015). Studies from both animal and human have suggested divergences in the gut microbiome composition result in different weight gain post same diet (Le Chatelier et al. 2013; Thaiss et al. 2016). In addition, some gut microbes may stimulate chronic low-grade inflammation by such as producing lipopolysaccharides, thereby contributing to obesity and insulin resistance (Boulange et al. 2016). Moreover, modulation of the gut microbiota by fiber supplementation or fecal microbiota transplantation can suppress inflammation and improve insulin sensitivity, suggesting the vital role of the gut microbiota in etiology of metabolic syndrome (Cani et al. 2008).

Investigations have been performed to identify the gut microbiota markers of obesity; However, no consistent pattern was not obtained from different studies. For example, both Bäckhed et al. (Backhed et al. 2004) and Turnbaugh et al (Turnbaugh et al. 2006). found that a decreased ratio of Bacteroidetes to Firmicutes in people with obesity, but failed to be supported by the other human studies (Shi et al. 2006; Duncan et al. 2008). 
Indeed, the gut microbiome composition appears to be shaped by host genetics, age,

70

71 gender, geographical locations, and other environmental factors. By characterizing the gut microbiota of 7,009 individuals from 14 districts within the Guangdong province of China, He et al. showed that host location is the strongest explanatory factor to the microbiota variations (He et al. 2018). Our previous study demonstrated BMI differences in the gut microbiota are gender specific (Gao et al. 2018). Thus, the region, age and gender should be considered when choosing controls to compare the gut microbiome of disease cases.

By accounting for the geographical location and age as confounding factors, this study characterizes the composition and functions of the gut microbiota in obese Shenzhen adolescents, and evaluated the influence of gender on the BMI-related differences in the gut microbiome. (1)

(1)

\section{MATERIAL AND METHODS}

\section{Study cohort}

The study began following approval from the Institutional Review Board of the Shenzhen Institutes of Advanced Technology, Chinese Academy of Sciences (SIATIRB-131115-H0032), and was registered with ClinicalTrials.gov (number NCT02539836). All individuals participating in the study received informed consent from their guardians. Two hundred and seventeen Chinese adolescents were recruited 9 at Shenzhen Children's Hospital, between September and December 2015. Written 
90 informed consent was obtained from parents of the subjects before participation.

91 Subjects with any of the criteria below were excluded from the present study:

1. Type 1 or type 2 diabetes.

2. Antibiotic use in the two months prior to sampling.

3. Long-term use of medication (e.g. antihypertensive drugs).

4. Diarrhea.

5. Long-term constipation.

According to the WHO BMI-for-age percentile growth charts (growth reference 5-19 years; https://www.who.int/growthref/who2007_bmi_for_age/en/), we classified each participant into normal weight $(\mathrm{N})$, overweight $(\mathrm{OW})$, or obese $(\mathrm{OB})$. General characteristics of the subjects, body composition, and serological test results for each BMI group are shown in Table 1.

\section{Sample collection, DNA extraction, and 16S rRNA gene Sequencing}

Venous blood samples were collected at Shenzhen Children's Hospital upon annual physical examination. Serological assays were performed to determine white blood cell (WBC), red blood cell (RBC), Hemoglobin (Hb), proinsulin-like component (PLC), alanine aminotransferase (ALT), total bilirubin (TBIL), total protein (TP), albumin (ALB), Cerutoplasmin (CER/CP), ceruloplasmin (CRP), blood urea nitrogen (BUN), creatinine $(\mathrm{Cr})$, urine acid (UA), creatine kinase (CK), creatine kinase- myoglobin (CK$\mathrm{MB}$ ), insulin (INS), C-peptide, triglycerides (TG), total cholesterol (TC), high-density lipoprotein (HDL), low-density lipoprotein (LDL), apolipoprotein A1 (Apo-A1), apolipoprotein B (Apo-B), and polyunsaturated fatty acid (PUFA). 
112 Each participant donated $\sim 10 \mathrm{~g}$ of fresh stool and placed inside a sterile plastic bag with

113 ice pack. These samples were homogenized to a uniform consistency, and DNA was

114 routinely extracted from $0.3 \mathrm{~g}$ fecal material using TIANamp Stool DNA Kit

115 (TIANGEN BIOTECH, cat. \#DP328-02, Beijing, China), following the manufacturer's

116 instructions. DNA was quantified using a dsDNA HS assay on a Qubit 3.0 (Thermo

117 Fisher Scientific, USA). The universal primers (forward: 5'-AYTGGGYDTAAAGNG-

1183 ', reverse: 5'-TACNVGGGTATCTAATCC-3') were used to PCR amplify the isolated

119 genomic DNA for the V3-V4 16S rDNA hypervariable regions. The PCR products

120 were sequenced by an Illumina MiSeq (Illumina, Inc, San Diego, CA) using the 2x300

121 bp paired-end protocol.

122 16S rRNA Gene Sequencing Analysis

123 The raw sequencing data was quality filtered and demultiplexed using QIIME 1.9.1

124 (Caporaso et al. 2010). Chimeric sequences were identified and removed using

125 UCHIME (Edgar et al. 2011). Two-hundred and five samples which passed the quality

126 control were incorporated into analysis. Operational taxonomic units (OTUs) were

127 clustered using a closed-reference picking protocol with the UCLUST algorithm based

128 on $97 \%$ nucleotide similarity. Microbial OTUs were annotated with the Greengenes

129 reference database 13_8. OTU counts were processed with total sum normalization

130 (TSS) followed by cumulative-sum scaling (CSS) by using Calypso (Zakrzewski et al.

131 2017). The relative abundances were $\log 2$ transformed to account for the non-normality

132 of taxonomic counts data. 
133 The biodiversity was measured by number of OTUs, Chao1 index, Shannon index, and

134 Inverse Simpson index. We performed Principal Coordinate Analysis (PCoA) to

135 determine whether the samples could be separated on the basis of BMI and gender.

136 Significance of composition difference among groups was determined using analysis

137 of variance (ANOVA) followed by Wilcoxon test for pairwise comparisons. We used

138 Spearman correlations to identify the BMI-associated taxa. We considered OTUs

139 appeared in more than $25 \%$ of the samples, that had significant correlation $(\mathrm{p}<0.01)$

140 with BMI. PICRUSt (Phylogenetic Investigation of Communities by Reconstruction of

141 Unobserved States) (Langille et al. 2013) was applied to obtain the KEGG (Kyoto

142 Encyclopedia of Genes and Genomes) pathways through predicting metagenome

143 content from 16S rRNA gene surveys.

144 A Random Forest (RF) model was constructed by using R-package randomForest

145 (Liaw and Wiener. 2002) to perform supervised classification of the three BMI

146 categories. Half of the dataset was used for building and training the RF model, and the

147 other half for testing. The optimal number of variables (mtry) randomly sampled as

148 candidates at each split was accessed by tuneRF function. We calculated the

149 interpolated area under the ROC curve (area under the receiver operating characteristic

150 curve, AUC) for each classifier based on the cross-validation testing results.

\section{RESULTS}

153 BMI Is Associated with Compositional Changes in The Gut Microbiome of 154 Adolescents 
155 After quality filtering, a total of 17,323,193 sequencing reads were obtained from 205

156 fecal samples. Taxa that have less than $0.01 \%$ relative abundance across all samples

157 were excluded. An overall of 518 OTUs were identified, which were grouped in 9

158 phylum and 69 genera. Richness (number of OTUs and Chao1 indexes) and alpha

159 diversity (Shannon and Inverse Simpson indexes) were estimated at the OTU level with

160 samples rarefied to depth of 37,027 reads (the lowest number of sequences). The

161 richness of was higher in the overweight subjects compared with those with normal

162 weight (Figure S1A, B). No significance in alpha diversity was obtained among the

163 three BMI groups (Figure S1C, D). With respect to beta diversity, PCoA of Bray-Curtis

164 dissimilarity index demonstrated that the overall gut microbiome composition was not

165 able to be stratified by BMI (Figure S2).

166 Differential taxa abundance was analyzed using one-way ANOVA. At the phylum level,

167 Verrucomicrobia was more abundant in obese adolescents (Figure 1A). At the family

168 level, obese adolescents had increased abundances of Actinomycetaceae (including

169 genera Actinobacteria and Collinsella; Figure 1C), Clostridiaceae (including genera

170 Clostridium and SMB53; Figure 1C), Cytophagaceae (including Rhodococcus genus;

171 Figure 1C), Streptococcaceae (including Streptococcus genus; Figure 1C), and

172 Veillonellaceae, and decreased abundances of Christensenellaceae and Rikenellaceae

173 (Figure 1B). The relative abundance of Nocardiaceae family was particular higher in

174 the overweight subjects. In addition, Erwinia belonging to Enterobacteriaceae family

175 was found with a higher relative abundance in obese adolescents (Figure 1C). 
176 Fifty-six taxa were identified with significant correlations with BMI with most of the

177 members belonging to the Firmicutes phylum (48 OTUs), and 17 and 12 taxa part of

178 the Ruminococcaceae and Lachnospiraceae families, respectively (Table S1, S2, S3).

179 For each content of serological surveys and boy composition, we used Spearman

180 correlation method to identify their associations with the 56 BMI-associated taxa. Three

181 Firmicutes taxa (two OTUs belonging to Ruminococcaceae and one belonging to

182 Lachnospiraceae) were found having relatively strong positive correlations with BMI,

183 body fate rate, waistline, and inverse correlations with body water, protein, muscle, and

184 mineral salts rates (Figure 2A). Notably, these three taxa also positively correlated with

185 most of serum biochemical parameters, except TBIL. Notably, a few of

186 Ruminococcaceae bacteria were inversely correlated with BMI, including

187 Faecalibacterium prausnitzii.

188 To further investigate the BMI-associated bacterial taxonomic biomarkers in the gut

189 microbiome in Chinese adolescents, we built a RF model to classify the phenotypes

190 based on the 56 OTUs identified by the Spearman correlation. Tuning of the RF model

191 resulted in $m$ try $=14$ for $n$ tree $=500$. The 30 most BMI-discriminatory taxa identified by

192 the RF model were shown in Figure 2B in rank order of their contribution to the

193 predictive accuracy. We calculated the interpolated area under the receiver operating

194 characteristic (ROC) curves (AUC) for the classifier based on the cross-validation

195 testing results. We successfully classified obese adolescents with a high classifiability

196 (Figure 2C; AUC=0.96). Adolescents with normal weight and those overweight were 
197 also able to be classified, with AUC of 0.86 and 0.76 for subjects with normal weight

198 and overweight, respectively.

Gut Microbiota Gene Content Associated with PPAR Signaling and Adipocytokine

\section{Signaling Are Reduced in Obese Adolescents}

201 We also used Spearman correlations to identify the BMI-associated functional profile of microbial community 16S rRNA sequence data by using PICRUSt (Langille et al.

2013). We selected seven KEGG pathways that had significant correlation $(\mathrm{p}<0.01)$

with BMI (Table S4, S5, S6). For each of the KEGG pathways that significantly associated with BMI, we further used Spearman correlations to identify their

207 predicted gene content of related to peroxisome, PPAR signaling pathway, and 208 adipocytokine signaling pathway showed inverse associations with BMI, body fat rate, and waistline, and positive correlation with body water rate, protein rate, muscle rate,

210 and mineral salts rate. In particular, PPAR signaling pathway and adipocytokine

211 signaling pathway were found significantly downregulated in the obese adolescents

212 comparing to the normal weight and overweight counterparts (Figure 3B). Additionally,

213 these pathways were inversely correlated with CER/CP, blood sugar level, (both systolic and diastolic) blood pressure, CRP, WBC, PUFA, and ALT. The predicted gene

215 content related to synthesis and degradation of ketone bodies, beta Alanine metabolism,

216 glycosyltransferases, and other ion coupled transporters positively associated with BMI, 217 body fat rate, and waistline, but negatively associated with body water rate, protein rate, 218 muscle rate, and mineral salts rate. 
With the gut microbiota sequencing data of 516 Chinese adult, we previously found that BMI differences in the gut microbiome composition are gender specific (Gao et al. 2018). Since age has also been reported as a confounding factor influencing the gut microbiome, and it is uncertain whether the gender differences discovered in adult people also exist in adolescents. Here we evaluate whether BMI differences in the gut microbiome of adolescents are influenced by gender. Neither alpha diversity nor beta diversity was found significantly different between Chinese boys and girls (Figure S1,

Clostridiaceae, Lachnospiraceae, Planococcaceae, and Streptococcaceae were more abundant in boys than girls (Figure S3A).

230 We then compared the relative abundances of the gut microbiome among the three BMI 231 categories with gender stratification (Figure S3B). At the family level, a higher 232 abundance of Cytophagaceae was observed in both obese girls and boys. Overweight 233 boys had a lower abundance of Actinomycetaceae, and a higher abundance of 234 Nocardiaceae than those with normal weight as well as obesity. At the genus level, 235 both obese boys and girls had higher relative abundances of Hymenobacter and 236 Megasphaera. A lower relative abundance of Odoribacter was particularly found in the 237 gut microbiome of obese boys. In addition, Actinomyces and Rhodococcus were 238 significantly lower and higher in overweight boys, respectively.

239 PICRUSt analysis revealed a higher level of bisphenol degradation in boys. A reduced

240 level of tropane piperidine and pyridine alkaloid biosynthesis was only observed in 
241 obese boys (Figure S4). Obese girls had an increased level of selenocompound

242 metabolism, and decreased levels of adipocytokine signaling pathway and ethylbenzene

243 degradation. A lower level of PPAR signaling pathway was detected in both genders.

\section{DISCUSSION}

246 The identification of gut microbial signatures that are responsible of obesity has great

247 potential in prevention and treatment of overweight and obesity. Here we report BMI-

248 associated patterns in the adolescent gut microbiome composition and functions. The

249 imbalance of Bacteroidetes to Firmicutes ratio has been reported in numerous studies

250 (Duncan et al, 2008; Jumpertz et al. 2011; Schwiertz et al. 2011), and we previously

251 reported that Bacteroidetes was enriched in obese adults comparing to lean subjects

252 (Gao et al. 2018). In the present data, no difference in the relative abundances of these

253 two phyla upon comparison of obese and normal-weight adolescents, which is in

254 agreement with findings of (Duncan et al. 2008; Jumpertz et al. 2011). We identified

25556 microbial taxa significantly $(\mathrm{p}<0.01)$ correlated with BMI, and a large proportion of

256 them belonging to Firmicutes phylum, especially families Ruminococcaceae and

257 Lachnospiraceae. Supervised learning algorithm constructed based on the 56 BMI-

258 associated OTUs resulted in the successful classification of obese adolescents with a

259 high accuracy exceeding $90 \%$.

260 The enrichment of Ruminococcaceae has been observed in animals fed by high-fat diet

261 (Schwiertz et al. 2011), but some Ruminococcaceae taxa were also linked to a lower

262 risk of weight gain (Menni et al. 2017). In human studies, higher abundances of 

associated with lower BMI (Jumpertz et al. 2011; Goodrich et al. 2014). From our data, we identified 17 OTUs assigned to Ruminococcaceae were significantly correlated with BMI, and many of which were inversely correlated with BMI including the well-studied

269 butyrate producer Faecalibacterium prausnitzii, which is consistent with several 270 studies (Borgo et al. 2018; Tilg and Moschen. 2014). Butyrate has been known exerting 271 a profound effect in regulating metabolic inflammation, and reduced level of butyrate 272 may contribute to low-grade chronic inflammation that participating the development 273 of obesity. Nevertheless, there were also some Ruminococcaceae members positively 274 correlated with BMI. Thus, investigations at higher phylogenetic level are needed to 275 identify the specific bacteria species in terms of influence in weight gain.

276 Animal studies demonstrated that the abundance of family Lachnospiraceae increased 277 along with body weight of mice fed with high-fat diet (Ravussin et al. 2012); 278 colonization of Lachnospiraceae in germ-free mice induced significant increases in 279 fasting blood glucose concentrations as well liver and mesenteric adipose weights, and 280 reductions in plasma insulin levels. A human study with 190 Mexican children showed 281 that the family Lachnospiraceae was significantly increased in overweight and obese 282 children (Murugesan et al. 2015). Taken together, Lachnospiraceae may play a pivotal 283 role in the development of obesity and type 2 diabetes (Kameyama et al. 2014). From 284 our data, significant associations were obtained between 12 taxa of Lachnospiraceae 

and negative (4 OTUs) correlations were detected between BMI and the

287 Lachnospiraceae taxa. Hence, the specific species and strains are needed to be 288 determined in terms of their influences in blood glucose level and weight gain.

289 Although the exact mechanisms by which the gut microbiota contributes to obesity are

290 unclear, it is well established that modification of the gut microbiota can increase 291 energy production, trigger low-grade inflammation, induce insulin resistance, and 292 affect fatty acid tissue composition (Musso et al. 2011). Based on the inferred functional 293 profiles of the fecal microbiome, we found that genes associated PPAR and 294 adipocytokine signaling pathways were inversely correlated with CER/CP, blood sugar 295 level, (both systolic and diastolic) blood pressure, CRP, WBC, PUFA, and ALT, and 296 their levels were significantly downregulated in obese adolescents. PPARs are a 297 member of the nuclear receptor superfamily of ligand-dependent transcription factors 298 that is predominantly expressed in adipose tissue and the intestines, and it plays the 299 master role in adipogenesis for obesity development (Lefterova et al. 2014). Lower 300 levels of PPARs have been observed in obese patients, and activation of PPARs could 301 decrease fibro-inflammation and ectopic fat accumulation in the adipose tissue 302 (Corrales et al. 2018). Decline in bacterial genes capable of altering PPAR signaling 303 may reflect a reduction of PPARs expression in the host. In fact, some gut bacteria have 304 been found with capacities of regulating PPARs. For example, mice artificially infected 305 with Trichinella spiralis could induce a decrease in the levels of PPAR $\gamma$ in the colon, 306 which is accompanied by a decline in beneficial species (such as Akkermansia) and 
307 increase in pathogenic bacteria (such as Escherichia/Shigella) (Chen et al. 2017).

308 Another pathway of importance that was differentially decreased in the obese

309 adolescent was adipocytokine signaling. Adipocytokines derived from adipose tissue,

310 including various hormones (such as leptin, adiponectin, resistin, visfatin) and

311 cytokines (such as interleukin-6 and tumor necrosis factor $\alpha$ ), are important regulators

312 of energy homeostasis and mediators of inflammation and immunity (Tilg and Moschen.

313 2006). There is overwhelming evidence that deficiencies in adipocytokines contribute

314 to the development of obesity and the associated comorbidities (Cao. 2014).

315 Importantly, some adipocytokines are able to modulate gut microbial composition

316 independently of dietary (Rajala et al. 2014). Further investigations are needed to

317 identify the exact bacterial products that affect these two pathways in order to determine

318 their roles in adiposity.

319 In our previous study with the fecal microbiome profiles Chinese adults (527 adults

320 aged $37.3 \pm 16.3$ ), enrichment of Fusobacteria and Actinobacteria were observed in the

321 male and female obese subjects, respectively (Gao et al. 2018). Functionally, bacterial

322 genes associated with butyrate-acetoacetate CoA-transferase were found to be enriched

323 in the gut microbiome of obese Chinese adults (Gao et al. 2018). However, these

324 features were not obtained from the adolescent data (205 adolescents aged $13.31 \pm 1.55$ ).

325 These inconsistencies may relate to differences of age. The influence of gender on the

326 gut microbiota have also been investigated in studies from ours and others. For example,

327 members of Bacteroides were found in a lower level in adult females than males from

328 surveys of European (Mueller et al. 2006) and US populations (Dominianni et al. 2015), 
329 which was also observed from our data of Shenzhen adolescents, but not in our previous

330 study with Chinese adults (Gao et al. 2018). Indeed, geographical location has been

331 found exerting a strong effect on human gut microbiota variations (He et al. 2018).

332 Shenzhen is one of the most developed cities in China and world-wide, and its lifestyles

333 (especially diet) are much similar to western countries than other areas of China. Hence,

334 some similarities the gut microbiome composition may exist between Shenzhen citizens

335 and westerners. Taken together, the influence of geographical location, age, and gender

336 can (partly) explain the inconsistent patterns across studies.

337 In conclusion, the BMI-associated differences in gut microbiota profiles were able to

338 be used as biomarkers for characterizing obese adolescents. The gene contents

339 associated with PPAR signaling pathway significantly reduced in the gut microbiome

340 of obese adolescents, and determining the exact metabolites produced by specific

341 species may provide invaluable microbial targets for prevention, assessment, and

342 treatment of obesity for adolescents. Our result reinforced a need to consider the

343 influence of age, gender and geographical location when choosing controls to compare

344 the gut microbiome of disease cases.

\section{DATA AVAILABILITY}

347 Raw sequencing data have been deposited on the European Nucleotide Archive server, 348 with accession number PRJEB33385. 
351 We acknowledge the contributions of the nursing staff of Shenzhen Children's Hospital

352 for their help with blood, stool, and metadata collection. We thank all volunteers for

353 their participation in this study.

\section{AUTHOR CONTRIBUTIONS}

356 YL designed the study; BW, YP, MZ and SZ collected the samples; YP performed the

357 experiment; XG and YC analyzed the data; XG, BW, YL and YC prepared the original 358 draft; all authors read and approved the final version of the manuscript.

\section{COMPLIANCE WITH ETHICAL STANDARDS}

361 Funding: This study was funded by the Shenzhen key technology R\&D program 362 (JSGG20170413152936281，20170502165510880), and University of Electronic

363 Science and Technology of China (Y03019023601008022).

364 Conflict of Interest: All author declare that he/she has no conflict of interest.

365 Ethical approval:

366 All procedures performed in studies involving human participants were in accordance 367 with the ethical standards of the institutional and/or national research committee and 368 with the 1964 Helsinki declaration and its later amendments or comparable ethical 369 standards. 
Backhed F., Ding H., Wang T., Hooper L.V., Koh G.Y., Nagy A., Semenkovich C.F., Gordon J.I. (2004). The gut microbiota as an environmental factor that regulates fat storage. Proc Natl Acad Sci U S A. 101, 15718-15723. doi: 10.1073/pnas.0407076101

Borgo F., Garbossa S., Riva A., Severgnini M., Luigiano C., Benetti A., Pontiroli A.E., Morace G., Borghi E.. (2018). Body Mass Index and Sex Affect Diverse Microbial Niches within the Gut. Front Microbiol. 9:213. doi: 10.3389/fmicb.2018.00213

Boulange C..L, Neves A.L., Chilloux J., Nicholson J.K., Dumas M.E.. (2016). Impact of the gut microbiota on inflammation, obesity, and metabolic disease. Genome Med. 8:42. doi: 10.1186/s13073-016-0303-2

Cani P.D., Bibiloni R., Knauf C., Waget A., Neyrinck A.M., Delzenne N.M., Burcelin R.. (2008). Changes in gut microbiota control metabolic endotoxemia-induced inflammation in high-fat diet-induced obesity and diabetes in mice. Diabetes. 57, 1470-1481. doi: 10.2337/db07-1403

Cao H.. (2014). Adipocytokines in obesity and metabolic disease. J Endocrinol. 220, T47-59. doi: 10.1530/JOE-13-0339

Caporaso J.G., Kuczynski J., Stombaugh J., Bittinger K., Bushman F.D., Costello E.K., Fierer N., Peña A.G., Goodrich J.K., Gordon J.I., Huttley G.A., Kelley S.T., Knights D., Koenig J.E., Ley R.E., Lozupone C.A., McDonald D., Muegge B.D., Pirrung M., Reeder J., Sevinsky J.R., Turnbaugh P.J., Walters W.A., Widmann J., Yatsunenko T., Zaneveld J., Knight R.. (2010). QIIME allows analysis of high-throughput community sequencing data. Nat Methods. 7, 335336. doi: $10.1038 /$ nmeth.f.303

Chen Q., Ren Y., Lu J., Bartlett M., Chen L., Zhang Y., Guo X., Liu C.. (2017). A Novel Prebiotic Blend Product Prevents Irritable Bowel Syndrome in Mice by Improving Gut Microbiota and Modulating Immune Response. Nutrients. 9, pii: E1341 doi: 10.3390/nu9121341

Collaboration NCDRF. (2017). Worldwide trends in body-mass index, underweight, overweight, and obesity from 1975 to 2016: a pooled analysis of 2416 population-based measurement studies in 128.9 million children, adolescents, and adults. Lancet. 390, 2627-2642. doi: 10.1016/S0140-6736(17)32129-3

Corrales P., Vidal-Puig A., Medina-Gomez G.. (2018). PPARs and Metabolic Disorders Associated with Challenged Adipose Tissue Plasticity. Int J Mol Sci. 19, pii: E2124. doi: 10.3390/ijms19072124.

Cox L.M., Blaser M.J.. (2015). Antibiotics in early life and obesity. Nat Rev Endocrinol. 11, 182-190. doi: 10.1038/nrendo.2014.210

Dominianni C., Sinha R., Goedert J.J., Pei Z., Yang L., Hayes R.B., Ahn J.. (2015). Sex, body mass index, and dietary fiber intake influence the human gut microbiome. PLoS One. 10:e0124599. doi: 10.1371/journal.pone.0124599

Duncan S.H., Lobley G.E., Holtrop G., Ince J., Johnstone A.M., Louis P., Flint H.J.. (2008). Human colonic microbiota associated with diet, obesity and weight loss. Int J Obes (Lond). 32, 17201724. doi: 10.1038/ijo.2008.155

Edgar R.C., Haas B.J., Clemente J.C., Quince C., Knight R.. (2011). UCHIME improves sensitivity and speed of chimera detection. Bioinformatics. 27, 2194-2200. doi: 10.1093/bioinformatics/btr381

Gao X., Zhang M., Xue J., Huang J., Zhuang R., Zhou X., Zhang H., Fu Q., Hao Y.. (2018). Body Mass Index Differences in the Gut Microbiota Are Gender Specific. Front Microbiol. 9:1250. doi: 10.3389/fmicb.2018.01250 
Goodrich J.K., Waters J..L, Poole A.C., Sutter J.L., Koren O., Blekhman R., Beaumont M., Van Treuren W., Knight R., Bell J.T., Spector T.D., Clark A.G., Ley R.E.. (2014). Human genetics shape the gut microbiome. Cell. 159, 789-799. doi: 10.1016/j.cell.2014.09.053

He Y., Wu W., Zheng H.M., Li P., McDonald D., Sheng H.F., Chen M.X., Chen Z.H., Ji G.Y., Zheng Z.D., Mujagond P., Chen X.J., Rong Z.H., Chen P., Lyu L.Y., Wang X., Wu C.B., Yu N., Xu Y.J., Yin J., Raes J., Knight R., Ma W.J., Zhou H.W.. (2018). Regional variation limits applications of healthy gut microbiome reference ranges and disease models. Nat Med. 24, 1532-1535. doi: 10.1038/s41591-018-0164-x

Jumpertz R., Le D.S., Turnbaugh P.J., Trinidad C., Bogardus C., Gordon J.I., Krakoff J.. (2011). Energy-balance studies reveal associations between gut microbes, caloric load, and nutrient absorption in humans. Am J Clin Nutr. 94, 58-65. doi: 10.3945/ajcn.110.010132

Kameyama K., Itoh K.. (2014). Intestinal colonization by a Lachnospiraceae bacterium contributes to the development of diabetes in obese mice. Microbes Environ. 29, 427-430. doi: 10.1264/jsme2.ME14054

Kasai C., Sugimoto K., Moritani I., Tanaka J., Oya Y., Inoue H., Tameda M., Shiraki K., Ito M., Takei Y., Takase K.. (2015). Comparison of the gut microbiota composition between obese and nonobese individuals in a Japanese population, as analyzed by terminal restriction fragment length polymorphism and next-generation sequencing. BMC Gastroenterol. 15:100. doi: 10.1186/s12876-015-0330-2

Langille M.G., Zaneveld J., Caporaso J.G., McDonald D., Knights D., Reyes J.A., Clemente J.C., Burkepile D.E., Vega Thurber R.L., Knight R., Beiko R.G., Huttenhower C.. (2013). Predictive functional profiling of microbial communities using 16S rRNA marker gene sequences. Nat Biotechnol. 31, 814-821. doi: 10.1038/nbt.2676

Le Chatelier E., Nielsen T., Qin J., Prifti E., Hildebrand F., Falony G., Almeida M., Arumugam M., Batto J.M., Kennedy S., Leonard P., Li J., Burgdorf K., Grarup N., Jørgensen T., Brandslund I., Nielsen H.B., Juncker A.S., Bertalan M., Levenez F., Pons N., Rasmussen S., Sunagawa S., Tap J., Tims S., Zoetendal E.G., Brunak S., Clément K., Doré J., Kleerebezem M., Kristiansen K., Renault P., Sicheritz-Ponten T., de Vos W.M., Zucker J.D., Raes J., Hansen T., MetaHIT consortium, Bork P., Wang J., Ehrlich S.D., Pedersen O.. (2013). Richness of human gut microbiome correlates with metabolic markers. Nature. 500, 541-546. doi: 10.1038/nature12506

Lefterova M.I., Haakonsson A.K., Lazar M.A., Mandrup S.. (2014). PPARgamma and the global map of adipogenesis and beyond. Trends Endocrinol Metab. 25, 293-302. doi: 10.1016/j.tem.2014.04.001

Ley R.E., Turnbaugh P.J., Klein S., Gordon J.I.. (2006). Microbial ecology: Human gut microbes associated with obesity. Nature. 444, 1022-1023. doi: 10.1038/4441022a

Liaw A., Wiener M.. (2002). Classification and Regression by randomForest. R News 23, 18-22.

Menni C., Jackson M.A., Pallister T., Steves C.J., Spector T.D., Valdes A.M.. (2017). Gut microbiome diversity and high-fibre intake are related to lower long-term weight gain. Int J Obes (Lond). 41, 1099-1105. doi: 10.1038/ijo.2017.66

Mueller S., Saunier K., Hanisch C., Norin E., Alm L., Midtvedt T., Cresci A., Silvi S., Orpianesi C., Verdenelli M.C., Clavel T., Koebnick C., Zunft H.J., Doré J., Blaut M.. (2006). Differences in fecal microbiota in different European study populations in relation to age, gender, and 
country: a cross-sectional study. Appl Environ Microbiol 72, 1027-1033. doi:

10.1128/AEM.72.2.1027-1033.2006

Murugesan S., Ulloa-Martinez M., Martinez-Rojano H., Galvan-Rodriguez F.M., Miranda-Brito C., Romano M.C., Pina-Escobedo A., Pizano-Zarate M.L., Hoyo-Vadillo C., Garcia-Mena J.. (2015). Study of the diversity and short-chain fatty acids production by the bacterial community in overweight and obese Mexican children. Eur J Clin Microbiol Infect Dis. 34, 1337-1346. doi: 10.1007/s10096-015-2355-4

Musso G., Gambino R., Cassader M.. (2010). Obesity, Diabetes, and Gut Microbiota: The hygiene hypothesis expanded? Diabetes Care. 33, 2277-2284. doi: 10.2337/dc10-0556

Rajala M.W., Patterson C.M., Opp J.S., Foltin S.K., Young V.B., Myers M.G. Jr.. (2014). Leptin acts independently of food intake to modulate gut microbial composition in male mice. Endocrinology. 155, 748-757. doi: 10.1210/en.2013-1085

Ravussin Y., Koren O., Spor A., LeDuc C., Gutman R., Stombaugh J., Knight R., Ley R.E., Leibel R.L.. (2012). Responses of gut microbiota to diet composition and weight loss in lean and obese mice. Obesity (Silver Spring). 20, 738-747. doi: 10.1038/oby.2011.111

Rios-Covian D., Ruas-Madiedo P., Margolles A., Gueimonde M., de los Reyes-Gavilan C.G., Salazar N.. (2016). Intestinal Short Chain Fatty Acids and their Link with Diet and Human Health. Front Microbiol. 7:185. doi: 10.3389/fmicb.2016.00185

Schwiertz A., Taras D., Schafer K., Beijer S., Bos N.A., Donus C., Hardt P.D.. (2010). Microbiota and SCFA in lean and overweight healthy subjects. Obesity (Silver Spring). 18, 190-195. doi: 10.1038/oby.2009.167

Shen J., Obin M.S., Zhao L.. (2013). The gut microbiota, obesity and insulin resistance. Mol Aspects Med. 34, 39-58. doi: 10.1016/j.mam.2012.11.001

Shi H., Kokoeva M.V., Inouye K., Tzameli I., Yin H., Flier J.S.. (2006). TLR4 links innate immunity and fatty acid-induced insulin resistance. J Clin Invest. 116, 3015-3025. doi: 10.1172/JCI28898

Thaiss C.A., Itav S., Rothschild D., Meijer M.T., Levy M., Moresi C., Dohnalová L., Braverman S., Rozin S., Malitsky S., Dori-Bachash M., Kuperman Y., Biton I., Gertler A., Harmelin A., Shapiro H., Halpern Z., Aharoni A., Segal E., Elinav E.. (2016). Persistent microbiome alterations modulate the rate of post-dieting weight regain. Nature. 540, 544-551. doi: 10.1038/nature20796

Tilg H., Moschen A.R.. (2006). Adipocytokines mediators linking adipose tissue, inflammation and immunity. Nat Rev Immunol. 6, 772-783. doi: 10.1038/nri1937

Tilg H., Moschen A.R.. (2014). Microbiota and diabetes: an evolving relationship. Gut. 63, 1513-1521. doi: 10.1136/gutjnl-2014-306928

Turnbaugh P.J., Ley R.E., Mahowald M.A., Magrini V., Mardis E.R., Gordon J.I.. (2006). An obesityassociated gut microbiome with increased capacity for energy harvest. Nature. 444, 10271031. doi: 10.1038/nature05414

Zakrzewski M., Proietti C., Ellis J.J., Hasan S., Brion M.J., Berger B., Krause L.. (2017). Calypso: a user-friendly web-server for mining and visualizing microbiome-environment interactions. Bioinformatics. 33, 782-783. doi: 10.1093/bioinformatics/btw725 
502 Table 1. Characteristics of the study population.

503

\begin{tabular}{|c|c|c|c|c|}
\hline Characteristics & Normal & Over Wight & Obesity & $p$-Value (ANOVA) \\
\hline Number (F/M) & $65(30 / 35)$ & $53(19 / 34)$ & $87(19 / 68)$ & \\
\hline Age (years) & $13.91 \pm 1.61$ & $13.26 \pm 1.50$ & $12.90 \pm 1.41$ & $2.75 \mathrm{E}-04$ \\
\hline Age Range (years) & $11-16$ & $11-16$ & $11-16$ & \\
\hline \multicolumn{5}{|l|}{ Anthropometric } \\
\hline Weight (kg) & $50.60 \pm 9.67$ & $62.88 \pm 8.85$ & $74.62 \pm 11.50$ & $2.99 \mathrm{E}-31$ \\
\hline Height (m) & $163.03 \pm 9.31$ & $162.81 \pm 8.94$ & $163.92 \pm 8.79$ & 7.34E-01 \\
\hline BMI & $18.87 \pm 1.96$ & $23.60 \pm 1.42$ & $27.65 \pm 2.53$ & $1.78 \mathrm{E}-63$ \\
\hline Waistline (cm) & $66.91 \pm 10.72$ & $78.19 \pm 6.81$ & $88.36 \pm 7.16$ & $9.40 \mathrm{E}-45$ \\
\hline \multicolumn{5}{|l|}{ Blood Pressure } \\
\hline Systolic blood pressure (mm Hg) & $114.28 \pm 14.48$ & $121.87 \pm 11.93$ & $125.76 \pm 12.48$ & $1.20 \mathrm{E}-06$ \\
\hline Diastolic blood pressure $(\mathrm{mm} \mathrm{Hg})$ & $70.78 \pm 8.17$ & $72.79 \pm 10.24$ & $76.59 \pm 8.74$ & $3.84 \mathrm{E}-04$ \\
\hline \multicolumn{5}{|l|}{ Body composition } \\
\hline Body fat & $7.67 \pm 4.12$ & $14.91 \pm 4.12$ & $21.81 \pm 10.60$ & $8.69 \mathrm{E}-40$ \\
\hline Body fat rate & $0.15 \pm 0.07$ & $0.23 \pm 0.08$ & $0.29 \pm 0.13$ & $6.94 \mathrm{E}-34$ \\
\hline Body muscle & $40.67 \pm 13.85$ & $45.08 \pm 14.78$ & $49.55 \pm 22.69$ & $2.95 \mathrm{E}-09$ \\
\hline Body muscle rate & $0.8 \pm 0.24$ & $0.71 \pm 0.21$ & $0.66 \pm 0.29$ & $8.49 \mathrm{E}-22$ \\
\hline Body mineral salts & $3.04 \pm 1.05$ & $3.66 \pm 1.18$ & $4.32 \pm 2.01$ & $1.62 \mathrm{E}-03$ \\
\hline Body mineral salts rate & $0.06 \pm 0.02$ & $0.06 \pm 0.02$ & $0.06 \pm 0.03$ & $8.19 \mathrm{E}-34$ \\
\hline Body water & $31.86 \pm 10.87$ & $35.10 \pm 11.48$ & $38.78 \pm 17.99$ & 4.09E-09 \\
\hline Body water rate & $0.62 \pm 0.19$ & $0.55 \pm 0.17$ & $0.51 \pm 0.23$ & $1.69 \mathrm{E}-27$ \\
\hline Body protein & $9.28 \pm 3.17$ & $9.98 \pm 3.30$ & $10.76 \pm 5.00$ & $1.62 \mathrm{E}-05$ \\
\hline Body protein rate & $0.18 \pm 0.06$ & $0.16 \pm 0.05$ & $0.14 \pm 0.06$ & $2.01 \mathrm{E}-31$ \\
\hline \multicolumn{5}{|l|}{ Serological investigations } \\
\hline ALB & $47.62 \pm 4.33$ & $46.86 \pm 3.99$ & $47.69 \pm 4.46$ & $5.06 \mathrm{E}-01$ \\
\hline ALT & $13.55 \pm 8.60$ & $17.06 \pm 12.79$ & $27.66 \pm 22.58$ & $1.26 \mathrm{E}-06$ \\
\hline ApoA1 & $1.38 \pm 0.17$ & $1.31 \pm 0.17$ & $1.33 \pm 0.17$ & $9.49 \mathrm{E}-02$ \\
\hline ApoB & $0.60 \pm 0.15$ & $0.64 \pm 0.16$ & $0.7 \pm 0.16$ & $1.91 \mathrm{E}-04$ \\
\hline BUN & $3.85 \pm 1.07$ & $3.95 \pm 0.86$ & $4.08 \pm 0.83$ & $3.03 \mathrm{E}-01$ \\
\hline $\mathrm{CER} / \mathrm{CP}$ & $24.35 \pm 4.64$ & $28.07 \pm 5.60$ & $29.06 \pm 6.39$ & $3.39 \mathrm{E}-06$ \\
\hline CK & $123.83 \pm 59.79$ & $133.43 \pm 68.69$ & $179.41 \pm 184.46$ & $2.00 \mathrm{E}-02$ \\
\hline CK-MB & $1.52 \pm 0.67$ & $1.60 \pm 0.80$ & $1.90 \pm 1.13$ & $3.35 \mathrm{E}-02$ \\
\hline C-peptide & $1.88 \pm 1.13$ & $2.48 \pm 1.80$ & $2.82 \pm 1.93$ & $3.47 \mathrm{E}-03$ \\
\hline $\mathrm{Cr}$ & $55.93 \pm 13.71$ & $54.46 \pm 11.00$ & $54.08 \pm 11.47$ & $6.32 \mathrm{E}-01$ \\
\hline CRP & $0.46 \pm 0.55$ & $1.39 \pm 1.91$ & $2.23 \pm 3.16$ & $2.94 \mathrm{E}-05$ \\
\hline $\mathrm{Hb}$ & $134.95 \pm 18.06$ & $137.30 \pm 11.03$ & $136.94 \pm 14.66$ & $6.35 \mathrm{E}-01$ \\
\hline HDL & $1.22 \pm 0.23$ & $1.12 \pm 0.23$ & $1.09 \pm 0.21$ & $1.18 \mathrm{E}-03$ \\
\hline INS & $10.35 \pm 8.00$ & $16.28 \pm 15.91$ & $22.04 \pm 17.67$ & $1.56 \mathrm{E}-05$ \\
\hline LDL & $2.34 \pm 0.56$ & $2.55 \pm 0.68$ & $2.69 \pm 0.55$ & $2.15 \mathrm{E}-03$ \\
\hline PLC & $291.12 \pm 64.73$ & $312.93 \pm 57.37$ & $329.56 \pm 63.03$ & $1.04 \mathrm{E}-03$ \\
\hline PUFA & $0.45 \pm 0.20$ & $0.50 \pm 0.21$ & $0.57 \pm 0.27$ & $6.06 \mathrm{E}-03$ \\
\hline $\mathrm{RBC}$ & $4.91 \pm 0.53$ & $4.90 \pm 0.36$ & $5.02 \pm 0.42$ & $1.94 \mathrm{E}-01$ \\
\hline TBIL & $9.85 \pm 4.52$ & $8.72 \pm 4.92$ & $7.23 \pm 3.11$ & $5.64 \mathrm{E}-04$ \\
\hline $\mathrm{TC}$ & $3.93 \pm 0.83$ & $4.07 \pm 0.85$ & $4.37 \pm 0.77$ & $3.47 \mathrm{E}-03$ \\
\hline TG & $1.09 \pm 0.60$ & $1.59 \pm 1.17$ & $1.76 \pm 0.94$ & $1.02 \mathrm{E}-03$ \\
\hline ТP & $76.90 \pm 7.98$ & $75.70 \pm 6.00$ & $76.41 \pm 5.50$ & $6.11 \mathrm{E}-01$ \\
\hline UA & $337.71 \pm 87.60$ & $398.79 \pm 120.42$ & $425.78 \pm 102.84$ & $2.55 \mathrm{E}-06$ \\
\hline WBC & $7.61 \pm 1.76$ & $8.60 \pm 2.05$ & $8.70 \pm 1.67$ & $6.79 \mathrm{E}-04$ \\
\hline
\end{tabular}

505 Values represent mean \pm standard error of the mean. 
508 Figure 1. Differences in fecal microbiome constituents of normal weight, overweight,

509 and obese Shenzhen adolescents. Bacterial taxa at the (A) family and (B) genus levels

510 demonstrated significantly different abundances across the three BMI groups. Each bar

511 plot indicates the mean proportion of sequences assigned to a feature in each group.

512 Whiskers represent $1.5^{*}$ inter-quartile range. Relative abundances were analyzed by

513 one-way ANOVA followed by Wilcoxon test for pairwise comparisons. **P $<0.01$ and

$514 * * * \mathrm{P}<0.001 . \mathrm{N}$, normal weight; OW, overweight; OB, obese.

516 Figure 2. Identifying BMI-associated bacterial taxonomic biomarkers Shenzhen

517 adolescents. (A) Heatmap of Spearman correlations between 54 BMI-associated

518 bacterial taxa and serum biochemical parameters and body composition. (B) Thirty

519 BMI-discriminatory bacterial taxa were identified by the RF model which were listed

520 in rank order of their contribution to the classification accuracy (mean decrease

521 accuracy). (C) ROC curve of the RF model.

523 Figure 3. Functional divergence of gut microbiota across different BMI groups.

524 (A) Predicted KEGG pathways that significantly correlated with BMI, and their 525 associations with the boy composition and serum biochemical parameters. (B) KEGG 526 pathways that are differentially expressed by the gut microbiome of the BMI categories. 527 Each bar plot indicates the mean proportion of sequences assigned to a feature in each 528 group. Whiskers represent $1.5^{*}$ inter-quartile range. Relative abundances of were 
bioRxiv preprint doi: https://doi.org/10.1101/745489; this version posted August 24, 2019. The copyright holder for this preprint (which was not certified by peer review) is the author/funder. All rights reserved. No reuse allowed without permission.

529 analyzed by one-way ANOVA followed by Wilcoxon test for pairwise comparisons.

$530 * * \mathrm{P}<0.01$ and $* * * \mathrm{P}<0.001 . \mathrm{N}$, normal weight; OW, overweight; OB, obese.

531 
A Actinomycetaceae

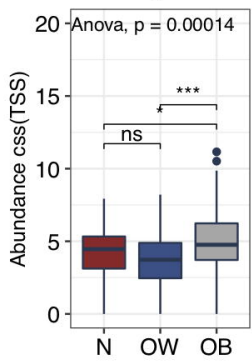

B Actinomyces

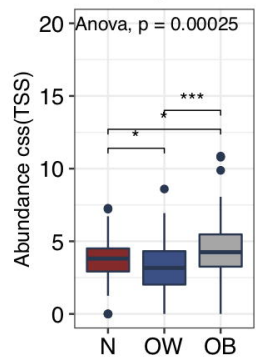

\section{Odoribacter}

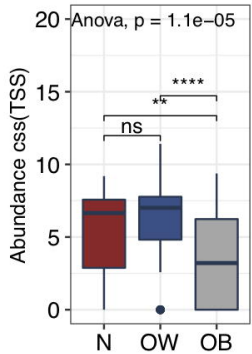

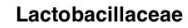

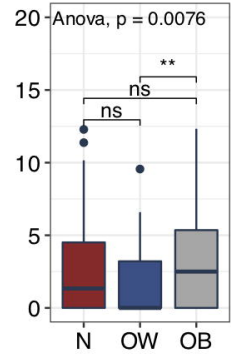

Clostridium

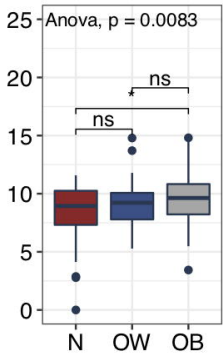

Rhodococcus

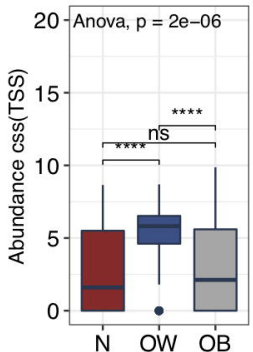

Rikenellaceae

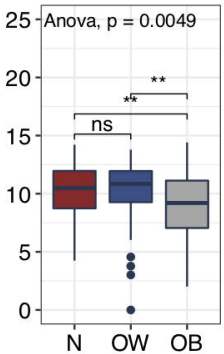

Erwinia

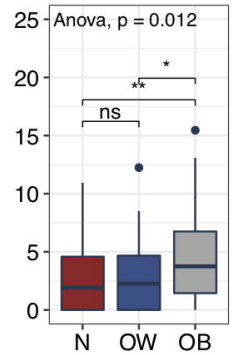

Streptococcus

$25-$ Anova, $p=0.0043$

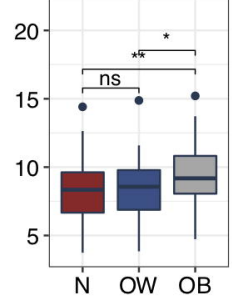

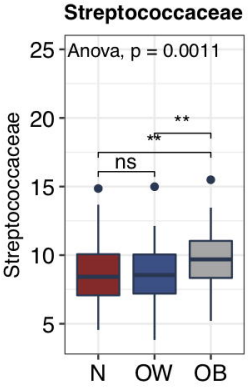

Hymenobacter

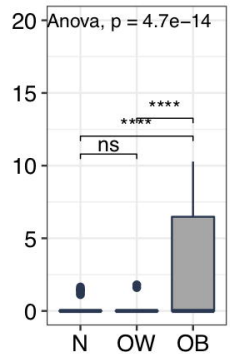

(U)Christensenellaceae

25 Anova, $p=0.0075$

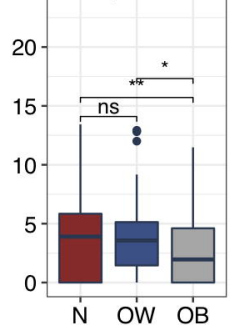

Veillonellaceae

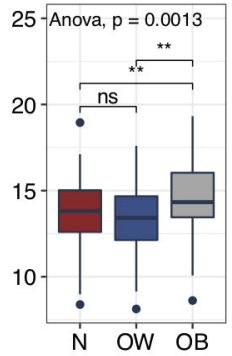

Lactobacillus

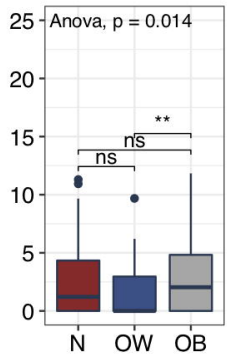

(U)Rikenellaceae

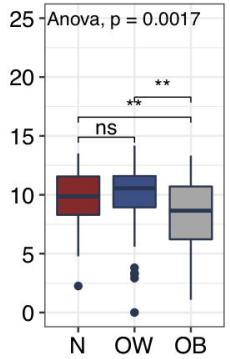

\section{Megasphaera}

25 -Anova, $p=6.2 e-05$

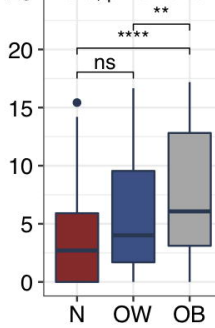




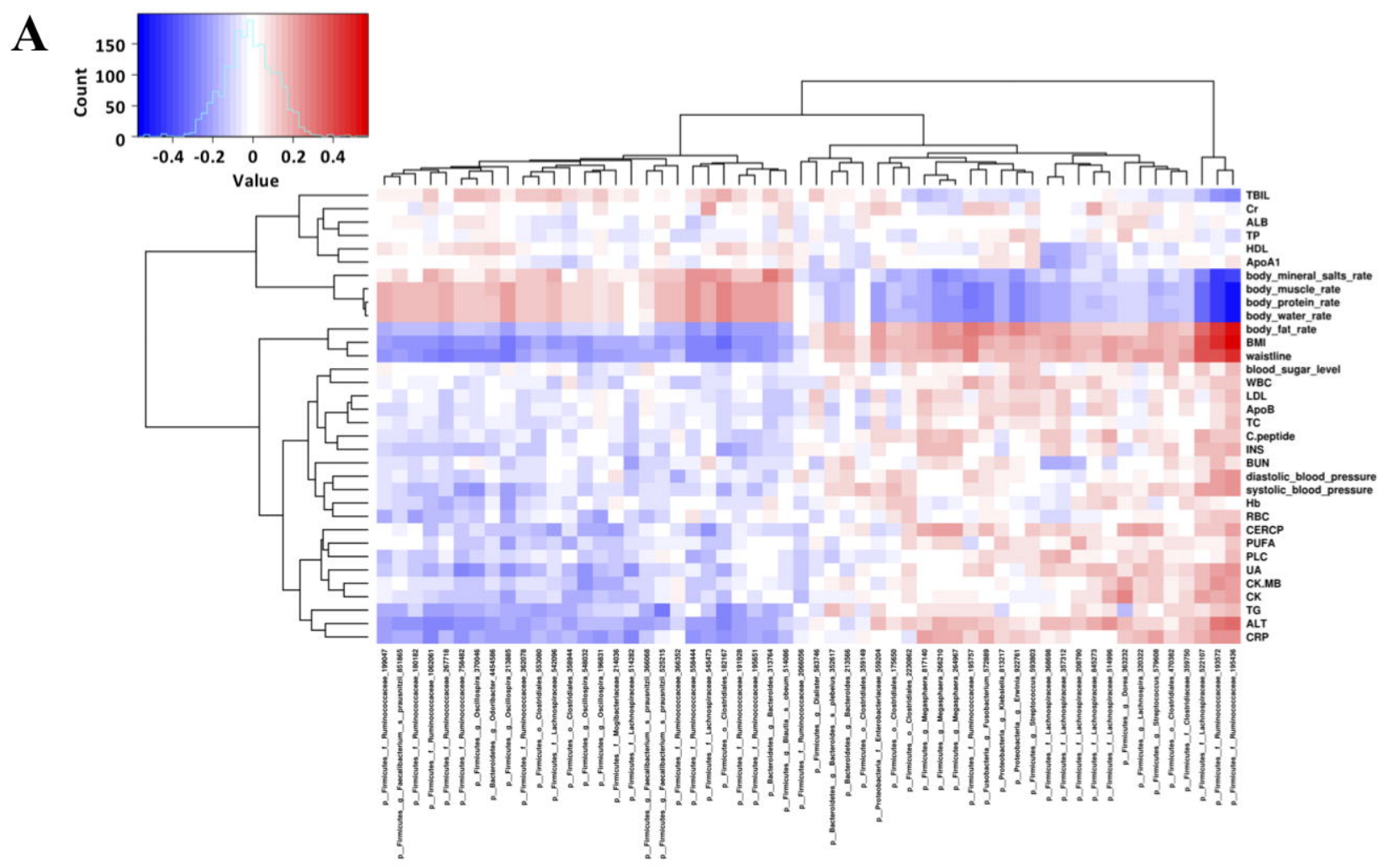

B
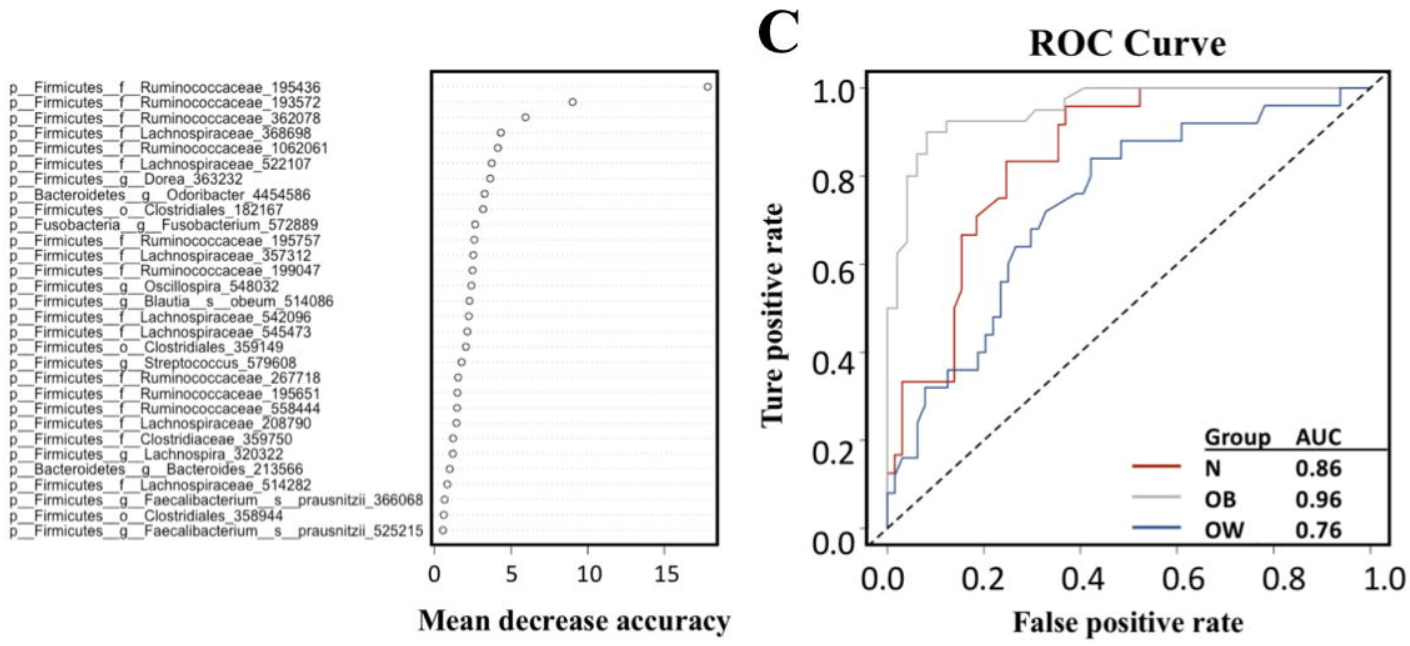


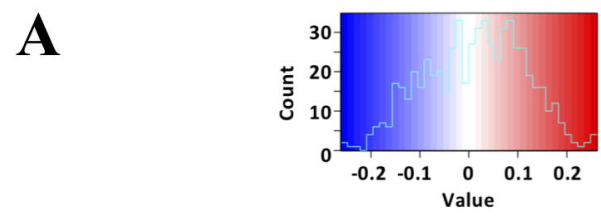

Peroxisome PPAR signaling pathway Adipocytokine signaling pathway Synthesis and degradation of ketone bodies beta Alanine metabolism Glycosyltransferases Other ion coupled transporters
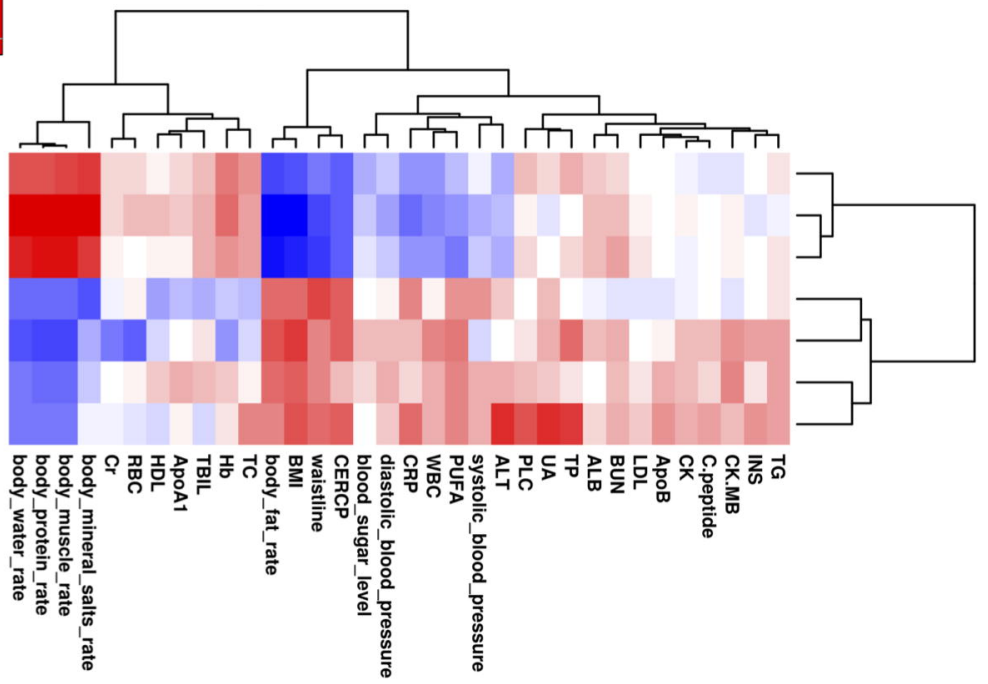

B
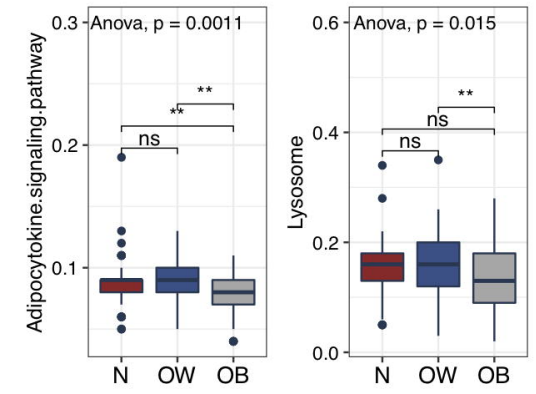
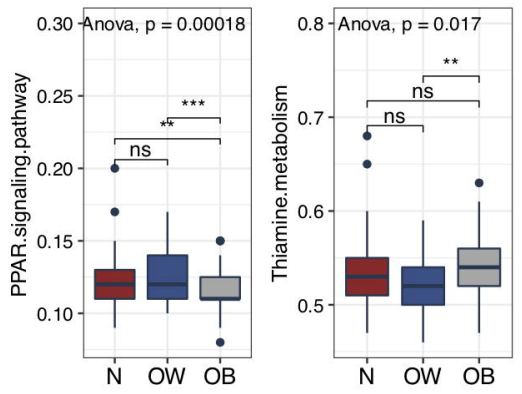